\author{
Tese De Doutorado \\ Universidade de SÃo Paulo
}

\title{
Efeitos em cascata em redes mutualistas
}

Marília Palumbo Gaiarsa

Agosto 2017

a 



\section{UniVERSIDADE DE SÃO PAUlO \\ Programa de Pós-GraduaÇÃo EM ECOlogia}

\section{EFEITOS EM CASCATA EM REDES MUTUALISTAS}

CASCADING EFFECTS IN MUTUALISTIC NETWORKS

\section{Marília Palumbo Gaiarsa}

Versão original da tese apresentada ao Instituto de Biociências da Universidade de São Paulo para a obtenção do título de Doutora em Ciências na área de Ecologia

Orientador: Prof. Dr. Paulo R. Guimarães Jr.

São Paulo

2017 



\section{Ficha catalográfica}

Gaiarsa, Marília Palumbo
Efeitos em cascata em redes mutualistas. Número de
páginas: 141 páginas
Tese (Doutorado)—Instituto de Biociências da Uni-
versidade de São Paulo. Departamento de Ecologia.
1. Interações ecológicas. 2. Mutualismo. 3. Redes
complexas. I. Universidade de São Paulo. Instituto de
Biociências. Departamento de Ecologia.

Comissão Julgadora:

Prof. Dr.

Prof. Dr.

Prof. Dr.

Prof. Dr.

Prof. Dr. Paulo R. Guimarães Jr.

(Orientador) 

Dedico essa tese ao melhor time do mundo, Papi, Mami e Pedro.

So don't be afraid. You hear me, young people? Don't be afraid. Be focused. Be determined. Be hopeful. Be empowered. Empower yourself with a good education. Then get out there and use that education to build a country worthy of you boundless promise. Lead by example with hope, never fear.

- Michele Obama

It is our choices, Harry, that show what we truly are, far more than our abilities.

- Albus Percival Wulfric Brian Dumbledore 



\section{Sumário}

Resumo . . . . . . . . . . . . . . . . . 15

Abstract . . . . . . . . . . . . . . . 17

Introdução geral . . . . . . . . . . . . . . . . . . . . . . . . . . . . . . . . . . . . . . . . .

Objetivos ......................... 25

Capítulo 1. Interaction strength promotes robustness in mutualistic

communities against cascading effects . . . . . . . . . . . . . 29

Introduction . . . . . . . . . . . . . . . . . . . . 33

Methods . . . . . . . . . . . . . . . 36

Results . . . . . . . . . . . . . . . . . 39

Discussion . . . . . . . . . . . . . . . . . . . 40

Figures . . . . . . . . . . . . . . . . . . 43

Capítulo 2. Robustness of ecological networks to multiple extinction

drivers ........................... 59

Introduction . . . . . . . . . . . . . . . . . . . . . . . . . . 63

Methods ...................... 66

Results . . . . . . . . . . . . . . . 70

Discussion . . . . . . . . . . . . . . . . . . . . . 72

Figures . . . . . . . . . . . . . . . . . . . 75

Capítulo 3. Opportunistic attachment assembles plant-pollinator networks .................... . 85

Introduction . . . . . . . . . . . . . . . . . . . . . . . . . 89

Methods . . . . . . . . . . . . . . . . . 92

Results . . . . . . . . . . . . . . . . . . . . . . . 98

Discussion . . . . . . . . . . . . . . . . . . 101

Figures . . . . . . . . . . . . . . . . . 106

Conclusões gerais . . . . . . . . . . . . . . . . . . . . . 123

Referências bibliográficas . . . . . . . . . . . . . . . . . 140 



\section{Resumo}

Espécies em comunidades ecológicas estão ligadas por meio de interações. Perturbações tais como flutuações em abundância, podem se propagar de uma espécie para outra espécie, por meio de interações ecológicas. Em consequência, perturbações podem percolar por toda comunidade e afetar todas as espécies, resultando em efeitos em cascata. Comunidades ecológicas diferem tanto em termos de composição de espécies quanto na maneira pela qual as interações estão organizadas dentro das comunidades. Como resultado, diferentes comunidades ecológicas são formadas por redes de interações com estruturas diversas, nas quais as espécies estão ligadas através de forças de interação que variam. Dado que espécies e interações estão sendo perdidas a ritmos alarmantes, é imprescindível compreender a robustez das diferentes comunidades frente a diferentes agentes promotores de extinção. Ademais, para que possamos prevenir o colapso das comunidades e restaurar interações perdidas, é necessário compreender como as comunidades são formadas, e como a robustez dessas comunidades muda com o passar do tempo. Entretanto, ainda não é claro como a estrutura da comunidade está relacionada com efeitos em cascata, e se as diferentes forças de interação entre as espécies afeta a robustez da rede, acelerando ou freiando os efeitos de diferentes promotores de extinção. Nesta tese, eu combinei simulações numéricas, redes teóricas e redes empíricas de interações mutualísticas para as quais existe informação sobre a força de interação entre espécies, para explorar a relação que estrutura e robustez têm com diferentes promotores de extinção e como a robustez dessas redes muda após a restauração de uma comunidade. Primeiramente eu investiguei como a estrutura da rede afeta o tempo até que uma perturbação atinja todas as espécies. O tempo de espalhamento foi usado como um indicador de robustez. Encontrei que as redes são mais robustas a efeitos em cascata quando incorporamos a força de interação das espécies, já que nestas simulações uma perturbação demorou mais tempo para atingir todas as espécies da rede. A riqueza de espécies, modularidade e aninhamento tiveram um forte efeito positivo no tempo de espalhamento da perturbação, independentemente da força de interação. Em seguida, criei redes teóricas com um número fixo de espécies e de interações, o que permitiu que eu isolasse os efeitos que o aninhamento e a modularidade têm na robustez. Explorei como a robustez a diferentes promotores de extinção está relacionada com o aninhamento e com a modularidade. Redes com valores maiores de aninhamento e 
modularidade apresentaram uma maior robustez a efeitos em cascata, enquanto que redes com valores intermediários de aninhamento foram mais robustas à remoção de espécies. Não encontrei nenhuma relação entre modularidade e remoção de espécies. Mostrei que a robustez depende não só do tipo de promotor de extinção que esta sendo avaliado, como da medida de interesse. Por fim, usando uma série temporal de oito anos de redes de planta a polinizadores após a restauração de um habitat na Califórnia (EUA) explorei como a montagem e robustez de comunidades muda com o passar do tempo. Encontrei que as posições que as espécies ocupam na rede são altamente dinâmicas com o passar do tempo, fazendo com que o processo de montagem de comunidades seja pontuado por grandes reorganizações da rede. Não encontrei uma relação entre robustez da rede tempo após a restauração. O conjunto de resultados apresentado e discutido nesta tese contribuí para o entendimento dos mecanismos subjacentes à manutenção da biodiversidade. Para compreendermos como a robustez de diferentes comunidades varia, é necessário considerar não só a espécie per se, mas também as espécies que dependem direta e indiretamente da espécie em questão, a estrutura da comunidade na qual aquela espécie está inserida e também da natureza da perturbação que atinge a comunidade. 


\section{Abstract}

Species in ecological communities are linked through interactions. Perturbations, such as fluctuations in abundance, can flow from a species to another through ecological interactions. As a consequence, perturbations can ripple across species assemblages resulting in cascading effects that can potentially affect all species in the community. Ecological assemblages differ both in terms of species composition and in the way in which interactions are organized. As a result, different ecological communities form interaction networks that differ both in their structures as well in the interaction strengths connecting pairs of species within networks. Given that species and interactions are being lost at alarming rates, it is imperative to comprehend how robust communities are to extinction drivers. Moreover, if we are to prevent communities' collapse and restore lost interactions, we have to understand how communities are assembled, as well as if and how robustness change through time. Despite continued effort by ecologists, it remains unclear how community structure is related to cascading effects and whether interaction strength affects network robustness by enhancing or dampening cascading effects due to multiple extinction drivers. In this thesis, I combine empirical data on weighted mutualistic networks, numerical simulations, and theoretical networks to explore how robust different network structures are to different extinction drivers, and how robustness change as networks assemble. First, I investigate how the structure of mutualistic networks affects perturbation spreading time - a proxy of network robustness to cascading effects. I found that networks are more robust to cascading effects when I incorporate interaction strengths, since simulations in which interaction strength was included had higher perturbation spreading times. Species richness, modularity, and nestedness had a strong, positive effect in perturbation spreading time regardless of the interaction strengths. Then, using theoretical networks with a fixed number of species and number of interactions, I was able to disentangle the effects nestedness and modularity have on robustness. I explore how robustness to different extinction drivers, in addition to cascading effects, is related to nestedness and modularity. Networks with greater nestedness and modularity were more robust to cascading effects, whereas networks with intermediate nestedness levels were the most robust to species removal. Modularity had no effect on robustness to species removal. Most importantly, I show that robustness depends not only on the type of extinction driver assessed, 
but also on the measure being used to quantify robustness. Finally, I use an eight-year dataset of plant-pollination networks following habitat restoration to explore how the assembly of plant-pollinator communities, and their robustness, changes as community assembles. I found that species occupy highly dynamic network positions through time, causing the assembly process to be punctuated by major network reorganizations. There was no relationship between years since restoration and robustness to perturbation spreading and to species removal. Altogether, these results contribute to broaden our understanding of the mechanisms behind biodiversity maintenance. If we are to protect and restore ecological communities, it is essential to consider not only the species per se, but also all species that depend directly and indirectly of that particular species, to unravel the relationship between network structure and community robustness to extinction drivers, and to understand how network structure and robustness changes as communities assemble. 


\section{Introdução geral}

You have to resign yourself to the fact that you waste a lot of trees before you write anything you really like, and that's just the way it is. It's like learning an instrument, you've got to be prepared for hitting wrong notes occasionally, or quite a lot, cause I wrote an awful lot before I wrote anything I was really happy with.

- J.K. Rowling 

Desde o trabalho clássico de Paine (1966) os ecólogos buscam entender qual o papel que cada espécie desempenha em comunidades ecológicas. Diversos estudos indicam que algumas espécies seriam especialmente importantes para a manutenção da estrutura da comunidade. A maior parte das evidências disponíveis indica a importância dos predadores de topo para a manutenção da diversidade (Paine, 1966; Estes \& Palmisano, 1974; Estes et al., 2011). Por exemplo, Estes \& Palmisano (1974) mostraram que a extinção local de populações de lontras marinhas (Enhydra lutris) na costa Oeste da América do Norte levou, em algumas localidades, a uma explosão populacional de uma de suas presas, o ouriço do mar (Strongylocentrotus sp.). O aumento populacional dos ouriços, por sua vez, levou à redução drástica de populações de kelps (Laminariales), espécies de algas marinhas que são consumidas pelos ouriços e que formam florestas subaquáticas na região. Como consequência do colapso das populações de kelps, a abundância de uma série de espécies mudou drasticamente. Desta forma, a extinção local de lontras levou à ocorrência de uma cascata trófica. Os resultados deste estudo ilustra a existência de efeitos em cascata, nos quais os efeitos de uma perturbação (e.g. extinção ou diminuição na abundância de uma espécie) se propagam através do sistema, atingindo não só as espécies que interagem diretamente com a espécie que sofreu a perturbação, mas potencialmente outras espécies do sistema (Paine, 1966; Estes \& Palmisano, 1974; Terborgh \& Estes, 2010; Estes et al., 2011). Especificamente, este tipo de efeito em cascata, na qual os efeitos de uma perturbação são alternados em diferentes níveis tróficos, é denominado cascata trófica.

Além de cascatas tróficas, trabalhos teóricos e estudos empíricos indicam a existência de outras formas de efeitos em cascata, como coextinções. Na coextinção a extinção de uma espécie leva à subsequente extinção de outras espécies que dependem diretamente da espécie extinta. Por exemplo, existem indícios de que há uma alta probabilidade de coextinção entre algumas espécies de plantas e seus polinizadores especialistas (Koh et al., 2004a; Anderson et al., 2011b) e de que a extinção de animais dispersores de sementes poderia levar à extinção de plantas que dependem desses animais para realizar a dispersão de suas sementes (Wotton \& Kelly, 2012). Ademais, coextinções devem ser ainda mais comuns em outros tipos de interações como por exemplo interações entre parasitas e hospedeiros (Dunn et al., 2009; Colwell et al., 2012).

Por fim, além de interações entre espécies, efeitos em cascata também podem influenciar negativamente processos ecossistêmicos (Estes et al., 2011; 
McCauley et al., 2012; Dirzo et al., 2014; Kaiser-Bunbury et al., 2017). Por exemplo, em um atol do Pacífico, McCauley et al. (2012) demonstraram empiricamente as consequências da substituição de vegetação nativa pelo plantio de palmeiras (Cocos nucifera) em algumas das ilhas. As ilhas que não possuiam vegetação nativa apresentaram uma diminuição na riqueza e uma mudança estrutural na vegetação, como também uma redução na deposição de nitrogênio no solo e no aporte de nutrientes para as comunidades marinhas ao redor das ilhas, resultando em uma menor densidade de raias manta (Manta birostris) nos arredores das ilhas devastadas. Desta forma, perturbações em uma espécie podem se propagar pela comunidade por diferentes rotas (e.g. Paine, 1966; Koh et al., 2004a; Estes et al., 2011; Colwell et al., 2012; McCauley et al., 2012) e influenciar diversas espécies, não só as que interagem com, ou dependem diretamente da, espécie perturbada.

Efeitos em cascata são, portanto, tanto causa quanto consequências da perda da biodiversidade (Young et al., 2016). Dado que estamos atualmente passando por um período de altas taxas de extinção (Barnosky et al., 2011; Dirzo et al., 2014; Ceballos et al., 2015), para que possamos atenuar os efeitos negativos da extinção de espécies é imprescindível que entendamos quais serão as consequências diretas e indiretas da diminuição na abundância e da perda de espécies. Entretanto, interações desaparecem antes mesmo que espécies sejam extintas (Janzen, 1974; Anderson et al., 2011b; Galetti et al., 2013; ValienteBanuet et al., 2015). Desta forma, é necessário que estratégias de conservação foquem não só na manutenção da biodiversidade, mas também na manutenção das interações ecológicas e dos serviços ecossistêmicos (McCann, 2007; Dirzo et al., 2014).

A perda da biodiversidade tem sido estudada principalmente por meio de estudos que consideram a extinção de espécies. Porém, dado o potencial para efeitos em cascata em comunidades ecológicas, é fundamental compreender como a estrutura advinda dos padrões de interações entre espécies está relacionada à estabilidade e robustez das comunidades ecológicas, para que possamos entender os mecanismos que geram, e mantém, a biodiversidade. A relação entre estabilidade e diversidade de sistemas ecológicos tem sido alvo de debate desde o trabalho clássico de May (1971), que demonstrou matematicamente que pode existir uma relação entre a estrutura da comunidade - a maneira na qual as interações entre espécies estão organizadas - e a dinâmica das comunidades (May, 1971; Pimm, 1984; Montoya et al., 2006; Allesina \& Tang, 2012). Espécies inte- 
ragem de diversas maneiras, de modo que as comunidades ecológicas podem ser vistas como redes nas quais diversas espécies estão interligadas por meio de diferentes tipos de interações, o "emaranhado de interações--entangled bank-ideia primeiramente proposta por Darwin (1859).

Uma das maneiras de se estudar interações entre espécies é por meio da abordagem de redes complexas. O estudo das redes complexas fornece um contexto teórico que permite avaliar as consequências das perturbações no nível da comunidade e dos ecossistemas, enfatizando a interação entre os organismos (Dunne et al., 2002; Bascompte \& Jordano, 2013). Na abordagem de redes ecológicas cada uma das espécies é representada por pontos, e a interação entre espécies é representada por linhas. A estrutura da rede pode ser descrita por uma miríade de métricas, que caracterizam como as interações estão distribuídas e organizadas entre as espécies. Algumas delas já foram relacionadas à robustez de redes ecológicas a perturbações, como a conectância, o aninhamento e a modularidade (Bascompte et al., 2003; Tylianakis et al., 2010; Stouffer \& Bascompte, 2011).

A conectância consiste na proporção de interações existentes, dentre todas as possíveis interações (Jordano, 1987) e já foi relacionada tanto ao aumento (Dunne et al., 2002; Lever et al., 2014) quanto à diminuição da robustez de redes (Thébault \& Fontaine, 2010). Já o aninhamento consiste em um padrão de interação no qual especialistas (espécies com poucas interações) interagem com conjuntos de espécies com as quais os generalistas (espécies com muitas interações) também interagem. Em redes aninhadas a perda de espécies especialistas parece não exercer resultar em nenhuma grande modificação na estrutura da rede, o que torna redes aninhadas robustas à extinção de espécies especialistas (Thébault \& Fontaine, 2010; Lever et al., 2014). Entretanto, dado que generalistas representam o cerne de redes aninhadas, a perda de espécies generalistas resulta em um rápido colapso de redes aninhadas (Tylianakis et al., 2010). Por fim, a modularidade consiste em subgrupos de espécies (módulos) que são pouco conectados entre si, mas que internamente possuem espécies fortemente conectadas (Newman \& Girvan, 2004; Lewinsohn et al., 2006; Olesen et al., 2007). Redes modulares parecem ser mais robustas dado que a propagação de efeitos em cascata parece ser mais lenta do que em redes não modulares (Tylianakis et al., 2010; Stouffer \& Bascompte, 2011; Allesina \& Tang, 2012, mas veja Fortuna et al. 2009). Entretanto, ainda não conhecemos a relação exata entre robustez de redes e os diferentes graus de conectância, aninhamento 
e modularidade que as redes apresentam (Tylianakis et al., 2010; Santamaría et al., 2016).

Uma das interações que tem sido muito estudada utilizando a ferramenta de redes complexas é o mutualismo. Mutualismos são interações interespecíficas na qual os indivíduos de ambas as espécies são beneficiados (Bascompte \& Jordano, 2013). Os mutualismos, como a polinização por exemplo, são interações interespecíficas que beneficiam os indivíduos de todas as espécies envolvidas. Existem diversos tipos de mutualismos, entre os quais a defesa de plantas contra herbivoria por formigas (e.g. Rico-Gray \& Oliveira, 2007), a dispersão de sementes por vertebrados (e.g. Donatti et al., 2011) e a remoção de parasitas por peixes limpadores de seus clientes (e.g. Sazima et al., 2010). Além conectância, aninhamento e modularidade, outro padrão comumente encontrado em redes mutualísticas é que existe uma assimetria nas interações (Bascompte et al., 2006; Vázquez et al., 2007; Fortuna et al., 2010), como nos casos em que um polinizador depende muito do recurso provido pela planta, mas planta não depende tanto do serviço prestado pelo animal. Mutualismos são interações ecológicas amplamente distribuídas e desempenham um papel fundamental na organização e na manutenção da biodiversidade, influenciando a ecologia e a evolução de comunidades ecológicas (Bascompte \& Jordano, 2007, 2013; Tylianakis, 2013).

Além de buscar entender a relação entre a robustez de comunidades e sua estrutura (May, 1971; Montoya et al., 2006; McCann, 2007; Allesina \& Tang, 2012; Tylianakis, 2013; Dirzo et al., 2014), alguns trabalhos visaram identificar se existem algumas espécies que seriam mais importantes na manutenção da estrutura da rede (e.g. Estes \& Palmisano, 1974; Saavedra et al., 2011; Vidal et al., 2014). Vidal et al. (2014) mostrou que as espécies de frugívoros que mais contribuíam para a estrutura da rede eram também as mais vulneráveis à extinção e Rezende et al. (2007b) mostraram que a perda de espécies não é aleatória, de modo que extinções podem desencadear cascatas de coextinções em espécies filogeneticamente próximas. Entretanto, sabemos que as interações ecológicas não são fixas, i.e., podem variar no tempo e no espaço, dependendo da disponibilidade de recursos, por exemplo. Desta forma, além de entender a importância das espécies na manutenção da estrutura da rede e das consequências ecológicas e evolutivas de sua perda (Rezende et al., 2007b; Galetti et al., 2013; Vidal et al., 2014), um passo importante é verificar como as interações variam no tempo. Por exemplo, Olesen et al. (2008) estudaram com ocorre a montagem 
de uma comunidade de plantas e polinizadores a partir do processo de sucessão primária e encontraram que novas espécies que chegam à comunidade tendem a interagir com espécies que já estavam previamente presentes, especialmente espécies generalistas. Portanto, entender como variam os padrões temporais e espaciais, e como ocorre a montagem das comunidades, é imprescindível para que possamos restaurar tanto comunidades devastadas quanto interações perdidas (Forup et al., 2008; Devoto et al., 2012). Kaiser-Bunbury et al. (2017) mostraram por exemplo, que áreas restauradas apresentaram não somente um aumento na diversidade de animais polinizadores, quanto na qualidade dos serviços de polinização.

A descrição dos padrões estruturais das redes ecológicas (Lewinsohn et al., 2006; Bascompte \& Jordano, 2007) foi seguida por estudos que avaliaram a robustez de redes ecológicas a diferentes cenários de remoções de espécies (e.g Memmott et al., 2004; Montoya et al., 2006; Vieira \& Almeida-Neto, 2015; Dunne et al., 2002). Por exemplo, redes mutualísticas parecem ser robustas à remoção aleatória de espécies, mas colapsam rapidamente se as espécies mais conectadas são removidas (Memmott et al., 2004; Dunne et al., 2002). Ademais, já é sabido que existe uma grande variação no quanto cada interação contribui para as dinâmicas populacionais (Lever et al., 2014). Recentemente, Vieira \& Almeida-Neto (2015) generalizaram o modelo clássico de remoção de espécies incluindo a frequência de interação entre espécies e a dependência de cada espécie em relação ao seu parceiro mutualista (e.g., quantas vezes um polinizador visitou uma planta, e quanto da dieta daquele polinizador depende do néctar fornecido por uma planta específica). Vieira \& Almeida-Neto (2015) encontraram que o número de extinções secundárias após a remoção de espécies foi inconsistente entre o modelo clássico e os resultados que eles obtiveram. Por fim, Santamaría et al. (2016) verificaram que a remoção de interações, ao invés de espécies, parece tornar as redes mutualísticas menos robustas e que a robustez das redes de interação parece ser muito dependente da abordagem utilizada. Nesse sentido, o próximo passo para ampliarmos nosso entendimento em relação à robustez de redes ecológicas é explorar como redes com diferentes estruturas variam em suas respostas a dieferentes efeitos em cascata.

\section{Objetivos}

Dado que a atual crise da biodiversidade representa uma ameaça não só às espécies per se como também à manutenção dos processos ecológicos e evo- 
lutivos das comunidades (Loreau et al., 2001; Estes et al., 2011; Colwell et al., 2012; McCauley et al., 2012; Tylianakis, 2013; Kaiser-Bunbury et al., 2017), é imprescindível compreender os mecanismos que modulam efeitos em cascata. Além disso, entender não só como perturbações se propagam em comunidades ecológicas, mas quais comunidades são mais vulneráveis a esses efeitos nos permite prever as respostas das comunidades frente à extinção das espécies (Dunne et al., 2002; Terborgh \& Estes, 2010). Desta forma, entender como perturbações em uma espécie afetam processos ecológicos é uma questão relevante frente às perturbações globais atuais (Tylianakis et al., 2010; Bascompte, 2009; Bracken \& Low, 2012; Kaiser-Bunbury et al., 2017).

O objetivo geral desta tese foi entender a relação entre estrutura de comunidades e robustez à promotores de extinção, além de ampliar o conhecimento atual em relação à variação temporal de comunidades. Assim, esta tese está organizada da seguinte forma: uma introdução geral, três capítulos ${ }^{1,2} \mathrm{em}$ forma de artigos e uma conclusão geral.

No PRIMEIRO capítulo criamos um modelo de simulação de efeitos em cascata para explorar qual a relação entre a padrões estruturais de redes mutualísticas e a velocidade de propagação de efeitos em cascata. Para isso, utilizamos dados oriundos de 17 redes empíricas e averiguamos como os resultados do modelo variavam quando consideramos redes binárias versus redes quantitativas. Encontramos que a assimetria de interação presente nas redes mutualísticas parece conferir robustez às redes, dado que perturbações demoraram significativamente mais tempo para se propagar por toda a rede após a incorporação da força de interação ${ }^{2}$.

No SEGUNDO capítulo criamos redes teóricas com riqueza e conectância fixas para explorar isoladamente o efeito da modularidade e do aninhamento na robustez das redes. Expandimos o modelo criado no primeiro capítulo e consideramos mais de um agente promotor de extinção agindo concomitantemente. Além disso, medimos a robustez da rede utilizandos três diferentes métricas: tempo de propagação de efeitos em cascata, quantidade de espécies secundariamente extintas e tempo até que a rede deixe de ser completamente conectada. Encontramos que a relação entre robustez e diferentes graus de aninhamento e modularidade varia de acordo com a métrica que está sendo avaliada, e que os resultados de mais de um promotor de extinção também dependem da métrica em questão.

\footnotetext{
${ }^{2}$ No momento da submissão da tese este capítulo está submetido para publicação.
} 
No TERCEIRO capítulo exploramos o processo de montagem de comunidades de plantas e polinizadores após a restauração. Para isso, utilizamos dados empíricos oriundos de uma série temporal de oito anos. Avaliamos como a estrutura e robustez da comunidade muda ao longo do tempo. As comunidades amostradas exibiram uma grande reorganização entre anos, tanto na composição de espécies, quanto no padrão de interação entre as espécies presentes. Entretanto, não encontramos nenhuma relação entre robustez e etempo após a restauração nessas comunidades ${ }^{3}$.

\footnotetext{
${ }^{3}$ Este capítulo encontra-se aceito para publicação no periódico Ecology Letters.
} 


\section{Conclusões gerais}

[...]No problem is too small or too trivial if we can really do something about it. You say you are a nameless man. You are not to your wife and to your child. You will not long remain so to your immediate colleagues if you can answer their simple questions when they come into your office. You are not nameless to me. Do not remain nameless to yourself-it is too sad a way to be. Know your place in the world and evaluate yourself fairly, not in terms of your naïve ideals of your own youth, nor in terms of what you erroneously imagine your teacher's ideals are.

Best of luck and happiness.

— Richard P. Feynman (carta escrita a Koichi Mano). 

Ao longo dos três capítulos que compõem esta tese abordei aspectos relacionados à organização de redes de interações ecológicas, focando especificamente em interações mutualísticas. Explorei os principais padrões estruturais encontrados e como estes padrões estão relacionados à robustez das redes, além de avaliar como a estrutura e robustez mudam ao longo do tempo.

No PRIMEIRO capítulo mostramos que existe uma possível relação entre a estrutura de redes mutualísticas e sua robustez a efeitos em cascata. A inclusão da força de interação nas simulações tornou as redes mais robustas à propagação de efeitos em cascata, e, contrário ao que é predito pelos modelos teóricos, não encontramos efeito da conectância na robustez à propagação de perturbações. Modularidade e aninhamento, ao contrário, possuem um forte efeito positivo que é ainda mais pronunciado quando consideramos a força de interação. Os resultados deste capítulo sugerem que, à medida que as espécies vão sendo extintas e a riqueza diminui, comunidades estarão cada vez mais susceptíveis à propagação de perturbações.

No SEGUNDO capítulo criamos redes teóricas para explorar os efeitos da modularidade e do aninhamento na robustez de redes ecológicas a diferentes promotores de extinção. Em todos os modelos de promotores de extinção avaliados, aninhamento esteve mais associado à robustez da rede do que modularidade. Além disso, não encontramos uma diferença significativa na resposta das redes teóricas aos diferentes modelos avaliados. Os resultados deste capítulo sugerem que combinações distintas de estrutura e dinâmica geram diferentes efeitos, e que não existe nenhuma resposta universal que relacione robustez e estrutura da rede.

No TERCEIRO capítulo mostramos que a montagem de uma comunidade de plantas e polinizadores na Califórnia (EUA) após a restauração se dá por um processo altamente dinâmico, pontuado por reorganizações tanto na composição de espécies, quanto na interação entre elas. Não encontramos nenhuma relação entre tempo após a restauração e as métricas de rede comumente utilizadas ou entre tempo após a restauração e robustez à remoção de espécies e efeitos em cascata. Além disso, sugerimos um novo mecanismo para explicar a montagem de comunidades, o anexo oportunista. Se resposta da comunidade à extinções de espécies for similar ao de montagem da comunidade, os resultados deste capítulo sugerem que comunidades que foram formadas por meio do anexo oportunista serão robustas à perda de espécies: à medida que espécies vão sendo extintas, as espécies remanescentes reorganizam suas interações, substituindo as espécies 
que não estão mais presentes e, desta forma, mantendo o serviço de polinização. Resta saber se o mecanismo do anexo oportunista é um padrão comum, ou particular às comunidades estudadas.

Em meus estudos futuros eu pretendo generalizar os modelos aqui explorados. Por exemplo, a maior parte dos estudos que abordam robustez de redes realizados até agora consideram que quando uma espécie perde todos os seus parceiros de interação, ela se torna secundariamente extinta. Entretanto, sabemos que isso não é verdade. Por exemplo, é sabido que espécies mudam suas interações ao longo. Desta forma, meus próximos passos incluem explorar como os resultados encontrados nos capítulos um e dois mudam quando incluímos a mudança nos padrões de interação observados no capítulo três.

Em conclusão, o presente estudo contribuí para o entendimento dos mecanismos subjacentes à manutenção da biodiversidade a à dinâmica de comunidades ecológicas ao longo do tempo. Adicionalmente, este estudo contribui para a discussão entre riqueza e robustez de comunidades ecológicas, ampliando o conhecimento dos processos que organizam interações ecológicas e sua relação com a propagação de efeitos em cascata, remoção de espécies e montagem de comunidades. De maneira geral, o conjunto de resultados apresentado e discutido aqui ressalta que, para compreendermos como varia a robustez de diferentes comunidades, é necessário considerar não só a espécie per se, mas também as espécies que dependem direta e indiretamente da espécie em questão, da estrutura da comunidade na qual aquela espécie está inserida e da natureza da perturbação que atinge a comunidade.

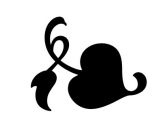




\section{Referências bibliográficas}

Ahn, Y.-Y., Bagrow, J. P. \& Lehmann, S. 2010. Link communities reveal multiscale complexity in networks. Nature, 466(7307):761-764.

Aizen, M. A., Sabatino, M. \& Tylianakis, J. M. 2012. Specialization and rarity predict nonrandom loss of interactions from mutualist networks. Science, 335 (6075):1486-1489.

Albrecht, M., Riesen, M. \& Schmid, B. 2010. Plant-pollinator network assembly along the chronosequence of a glacier foreland. Oikos, 119(10):1610-1624.

Allesina, S. \& Tang, S. 2012. Stability criteria for complex ecosystems. Nature, 483(7388):205-208.

Almeida-neto, M., Guimarães, P., Guimarães, P., Loyola, R. \& Ulrich, W. 2008. A consistent metric for nestedness analysis in ecological systems: reconciling concept and measurement. Oikos, 117:1227-1239.

Almeida-Neto, M., Guimarães, P., Guimarães, P. R., Loyola, R. D. \& Ulrich, W. 2008. A consistent metric for nestedness analysis in ecological systems: reconciling concept and measurement. Oikos, 117(8):1227-1239.

Anderson, M. J., Crist, T. O., Chase, J. M., Vellend, M., Inouye, B. D., Freestone, A. L., Sanders, N. J., Cornell, H. V., Comita, L. S., Davies, K. F. et al. 2011a. Navigating the multiple meanings of $\beta$ diversity: a roadmap for the practicing ecologist. Ecology Letters, 14(1):19-28.

Anderson, S. H., Kelly, D., Ladley, J. J., Molloy, S. \& Terry, J. 2011b. Cascading effects of bird functional extinction reduce pollination and plant density. Science, 331(6020):1068-1071.

Astegiano, J., Massol, F., Vidal, M. M., Cheptou, P.-O. \& Guimarães Jr, P. R. 2015. The robustness of plant-pollinator assemblages: linking plant interaction patterns and sensitivity to pollinator loss. PLoS ONE, 10(2):e0117243.

Atmar, W. \& Patterson, B. D. 1993. The measure of order and disorder in the distribution of species in fragmented habitat. Oecologia, 96(3):373-382.

Barabási, A.-L. \& Albert, R. 1999. Emergence of scaling in random networks. Science, 286(5439):509-512.

Barnosky, A. D., Matzke, N., Tomiya, S., Wogan, G. O., Swartz, B., Quental, T. B., Marshall, C., McGuire, J. L., Lindsey, E. L., Maguire, K. C. et al. 2011. Has the earth/'s sixth mass extinction already arrived? Nature, 471 (7336):51-57.

Bascompte, J. 2009. Disentangling the web of life. Science, 325(5939):416-419. 
Bascompte, J. 2010. Structure and dynamics of ecological networks. Science, 329(5993):765-766.

Bascompte, J. \& Jordano, P. 2007. Plant-animal mutualistic networks: the architecture of biodiversity. Annu. Rev. Ecol. Evol. Syst., 38:567-593.

Bascompte, J. \& Jordano, P. 2013. Mutualistic networks. Princeton University Press.

Bascompte, J. \& Stouffer, D. B. 2009. The assembly and disassembly of ecological networks. Phil. Trans. R. Soc. B, 364:1781.

Bascompte, J., Jordano, P., Melián, C. J. \& Olesen, J. M. 2003. The nested assembly of plant-animal mutualistic networks. Proceedings of the National Academy of Sciences, 100(16):9383-9387.

Bascompte, J., Jordano, P. \& Olesen, J. M. 2006. Asymmetric coevolutionary networks facilitate biodiversity maintenance. Science, 312(5772):431-433.

Bastolla, U., Fortuna, M. A., Pascual-García, A., Ferrera, A., Luque, B. \& Bascompte, J. 2009. The architecture of mutualistic networks minimizes competition and increases biodiversity. Nature, 458(7241):1018-1020.

Bates, D., Maechler, M., Bolker, B. \& Walker, S. lme4: Linear mixed-effects models using Eigen and $S_{4}, 2014$. R package version 1.1-7.

Blüthgen, N., Menzel, F. \& Blüthgen, N. 2006. Measuring specialization in species interaction networks. BMC Ecology, 6:9.

Blüthgen, N., Menzel, F., Hovestadt, T., Fiala, B. \& Blüthgen, N. 2007. Specialization, constraints, and conflicting interests in mutualistic networks. Curr. Biol., 17(4):341-346.

Blüthgen, N., Fründ, J., Vázquez, D. P. \& Menzel, F. 2008. What do interaction network metrics tell us about specialization and biological traits. Ecology, 89 (12):3387-3399.

Bracken, M. E. \& Low, N. H. 2012. Realistic losses of rare species disproportionately impact higher trophic levels. Ecology Letters, 15(5):461-467.

Brodie, J. F., Aslan, C. E., Rogers, H. S., Redford, K. H., Maron, J. L., Bronstein, J. L. \& Groves, C. R. 2014. Secondary extinctions of biodiversity. Trends in Ecology \& Evolution, 29(12):664-672.

Bronstein, J. L. 1994. Conditional outcomes in mutualistic interactions. Trends in Ecology \& Evolution, 9(6):214-217.

Brook, B. W., Sodhi, N. S. \& Bradshaw, C. J. 2008. Synergies among extinction drivers under global change. Trends in Ecology \& Evolution, 23(8):453-460. 
Brosi, B. J. 2016. Pollinator specialization: from the individual to the community. New Phytol., 210(4):1190-1194.

Brosi, B. J. \& Briggs, H. M. 2013. Single pollinator species losses reduce floral fidelity and plant reproductive function. Proceedings of the National Academy of Sciences, 110(32):13044-13048.

Burgos, E., Ceva, H., Perazzo, R. P., Devoto, M., Medan, D., Zimmermann, M. \& Delbue, A. M. 2007. Why nestedness in mutualistic networks? J. Theor. Biol., 249(2):307-313.

Cardinale, B. J., Duffy, J. E., Gonzalez, A., Hooper, D. U., Perrings, C., Venail, P., Narwani, A., Mace, G. M., Tilman, D., Wardle, D. A. et al. 2012. Biodiversity loss and its impact on humanity. Nature, 486(7401):59-67.

Ceballos, G., Ehrlich, P. R., Barnosky, A. D., García, A., Pringle, R. M. \& Palmer, T. M. 2015. Accelerated modern human-induced species losses: Entering the sixth mass extinction. Science Advances, 1(5):e1400253.

Chao, A., Chazdon, R. L., Colwell, R. K. \& Shen, T.-J. 2005. A new statistical approach for assessing similarity of species composition with incidence and abundance data. Ecology Letters, 8(2):148-159.

Chao, A., Colwell, R. K., Lin, C.-W. \& Gotelli, N. J. 2009. Sufficient sampling for asymptotic minimum species richness estimators. Ecology, 90(4):11251133.

Clauset, A., Moore, C. \& Newman, M. E. Structural inference of hierarchies in networks. In: Statistical network analysis: models, issues, and new directions, pp. 1-13. Springer, 2007.

Colwell, R. K., Dunn, R. R. \& Harris, N. C. 2012. Coextinction and persistence of dependent species in a changing world. Annual Review of Ecology, Evolution, and Systematics, 43:183-203.

Costa, L. d. F., Rodrigues, F. A., Travieso, G. \& Villas Boas, P. R. 2007. Characterization of complex networks: A survey of measurements. Advances in Physics, 56(1):167-242.

Csardi, G. \& Nepusz, T. 2006. The igraph software package for complex network research. InterJournal, pp. 1695.

Cuartas-Hernández, S. \& Medel, R. 2015. Topology of plant-flower-visitor networks in a tropical mountain forest: Insights on the role of altitudinal and temporal variation. PLoS ONE, 10(10):e0141804.

Darling, E. S. \& Côté, I. M. 2008. Quantifying the evidence for ecological synergies. Ecology Letters, 11(12):1278-1286. 
Darwin, C. 1859. On the origin of species by means of natural selection. 1859. London: Murray Google Scholar.

Dattilo, W. 2012. Different tolerances of symbiotic and nonsymbiotic ant-plant networks to species extinctions. Network Biology, 2(4):127.

de Sassi, C., Lewis, O. T. \& Tylianakis, J. M. 2012. Plant-mediated and nonadditive effects of two global change drivers on an insect herbivore community. Ecology, 93(8):1892-1901.

Devoto, M., Bailey, S., Craze, P. \& Memmott, J. 2012. Understanding and planning ecological restoration of plant-pollinator networks. Ecology Letters, $15(4): 319-328$.

Díaz-Castelazo, C., Guimarães Jr, P. R., Jordano, P., Thompson, J. N., Marquis, R. J. \& Rico-Gray, V. 2010. Changes of a mutualistic network over time: reanalysis over a 10-year period. Ecology, 91(3):793-801.

Dicks, L., Corbet, S. \& Pywell, R. 2002. Compartmentalization in plant-insect flower visitor webs. Journal of Animal Ecology, 71(1):32-43.

Dirzo, R. \& Raven, P. H. 2003. Global state of biodiversity and loss. Annual Review of Environment and Resources, 28(1):137-167.

Dirzo, R., Young, H. S., Galetti, M., Ceballos, G., Isaac, N. J. \& Collen, B. 2014. Defaunation in the anthropocene. Science, 345(6195):401-406.

Donatti, C. I., Guimarães, P. R., Galetti, M., Pizo, M. A., Marquitti, F. \& Dirzo, R. 2011. Analysis of a hyper-diverse seed dispersal network: modularity and underlying mechanisms. Ecology Letters, 14(8):773-781.

Dormann, C. F., Frueund, J., Bluethgen, N. \& Gruber, B. 2009. Indices, graphs and null models: analyzing bipartite ecological networks. The Open Ecology Journal, 2:7-24.

Dunn, R. R., Harris, N. C., Colwell, R. K., Koh, L. P. \& Sodhi, N. S. 2009. The sixth mass coextinction: are most endangered species parasites and mutualists? Proceedings of the Royal Society of London B: Biological Sciences, 276(1670):3037-3045.

Dunne, J. A. \& Williams, R. J. 2009. Cascading extinctions and community collapse in model food webs. Philosophical Transactions of the Royal Society $B, 364(1524): 1711-1723$.

Dunne, J. A., Williams, R. J. \& Martinez, N. D. 2002. Network structure and biodiversity loss in food webs: robustness increases with connectance. Ecology Letters, 5:558-567. 
Ehrlich, P. R. \& Daily, G. C. 1993. Population extinction and saving biodiversity. Ambio, pp. 64-68.

Ekstrom, C. MESS: Miscellaneous Esoteric Statistical Scripts, 2016. R package version $0.4-3$.

Emlen, J. M. 1968. Optimal choice in animals. Am. Nat., 102(926):385-389.

Estes, J. A. \& Palmisano, J. F. 1974. Sea otters: their role in structuring nearshore communities. Science, 185(4156):1058-1060.

Estes, J. A., Terborgh, J., Brashares, J. S., Power, M. E., Berger, J., Bond, W. J., Carpenter, S. R., Essington, T. E., Holt, R. D., Jackson, J. B. et al. 2011. Trophic downgrading of planet earth. Science, 333(6040):301-306.

Fiedler, M. 1973. Algebraic connectivity of graphs. Czechoslovak Mathematical Journal, 23(2):298-305.

Foley, J. A., DeFries, R., Asner, G. P., Barford, C., Bonan, G., Carpenter, S. R., Chapin, F. S., Coe, M. T., Daily, G. C., Gibbs, H. K. et al. 2005. Global consequences of land use. Science, 309(5734):570-574.

Folke, C., Holling, C. S. \& Perrings, C. 1996. Biological diversity, ecosystems, and the human scale. Ecological Applications, 6(4):1018-1024.

Fontaine, C., Guimarães, P. R., Kéfi, S., Loeuille, N., Memmott, J., van Der Putten, W. H., van Veen, F. J. \& Thébault, E. 2011. The ecological and evolutionary implications of merging different types of networks. Ecology Letters, 14(11):1170-1181.

Fort, H., Vázquez, D. P. \& Lan, B. L. 2016. Abundance and generalisation in mutualistic networks: solving the chicken-and-egg dilemma. Ecology Letters, 19(1):4-11.

Fortuna, M. A. \& Bascompte, J. 2006. Habitat loss and the structure of plantanimal mutualistic networks. Ecology Letters, 9(3):281-286.

Fortuna, M. A., Popa-Lisseanu, A. G., Ibáñez, C. \& Bascompte, J. 2009. The roosting spatial network of a bird-predator bat. Ecology, 90(4):934-944.

Fortuna, M. A., Stouffer, D. B., Olesen, J. M., Jordano, P., Mouillot, D., Krasnov, B. R., Poulin, R. \& Bascompte, J. 2010. Nestedness versus modularity in ecological networks: two sides of the same coin? Journal of Animal Ecology, $79(4): 811-817$.

Fortuna, M. A., Krishna, A. \& Bascompte, J. 2013. Habitat loss and the disassembly of mutalistic networks. Oikos, 122(6):938-942.

Forup, M. L., Henson, K. S., Craze, P. G. \& Memmott, J. 2008. The restoration 
of ecological interactions: plant-pollinator networks on ancient and restored heathlands. Journal of Applied Ecology, 45(3):742-752.

Freeman, L. C. 1978. Centrality in social networks conceptual clarification. Soc. Networks, 1(3):215-239.

Galeano, J., Pastor, J. M. \& Iriondo, J. M. 2009. Weighted-interaction nestedness estimator (wine): a new estimator to calculate over frequency matrices. Environ. Model. Softw., 24(11):1342-1346.

Galetti, M., Guevara, R., Côrtes, M. C., Fadini, R., Von Matter, S., Leite, A. B., Labecca, F., Ribeiro, T., Carvalho, C. S., Collevatti, R. G. et al. 2013. Functional extinction of birds drives rapid evolutionary changes in seed size. Science, 340(6136):1086-1090.

Garibaldi, L. A., Carvalheiro, L. G., Leonhardt, S. D., Aizen, M. A., Blaauw, B. R., Isaacs, R., Kuhlmann, M., Kleijn, D., Klein, A. M., Kremen, C. et al. 2014. From research to action: enhancing crop yield through wild pollinators. Frontiers in Ecology and the Environment, 12(8):439-447.

Gibert, J. P., Pires, M. M., Thompson, J. N. \& Guimarães Jr, P. R. 2013. The spatial structure of antagonistic species affects coevolution in predictable ways. The American Naturalist, 182(5):578-591.

Gilarranz, L. J., Rayfield, B., Liñán-Cembrano, G., Bascompte, J. \& Gonzalez, A. 2017. Effects of network modularity on the spread of perturbation impact in experimental metapopulations. Science, 357(6347):199-201.

Gómez, J. M. \& Zamora, R. 2006. Ecological factors that promote the evolution of generalization in pollination systems. Plant-pollinator interactions, N.M. Waser, J. Ollerton (eds.). The University of Chicago Press, Chicago, pp. $145-166$.

Grace, J. B., Schoolmaster, D. R., Guntenspergen, G. R., Little, A. M., Mitchell, B. R., Miller, K. M. \& Schweiger, E. W. 2012. Guidelines for a graph-theoretic implementation of structural equation modeling. Ecosphere, 3(8):1-44.

Graham, C. H. \& Fine, P. V. 2008. Phylogenetic beta diversity: linking ecological and evolutionary processes across space in time. Ecology Letters, 11 (12):1265-1277.

Guimarães Jr, P. R., Jordano, P. \& Thompson, J. N. 2011. Evolution and coevolution in mutualistic networks. Ecology Letters, 14(9):877-885.

Guimerá, R. \& Amaral, L. A. N. 2005. Cartography of complex networks: modules and universal roles. Journal of Statistical Mechanics: Theory and Experiment, 2005(02):P02001. 
Hagen, M., Kissling, W. D., Rasmussen, C., Carstensen, D., Dupont, Y., KaiserBunbury, C., O’Gorman, E., Olesen, J., De Aguiar, M., Brown, L. et al. 2012. Biodiversity, species interactions and ecological networks in a fragmented world. Advances in Ecological Research, 46:89-120.

Hooper, D. U., Adair, E. C., Cardinale, B. J., Byrnes, J. E., Hungate, B. A., Matulich, K. L., Gonzalez, A., Duffy, J. E., Gamfeldt, L. \& O'Connor, M. I. 2012. A global synthesis reveals biodiversity loss as a major driver of ecosystem change. Nature, 486(7401):105-108.

Jacobs, A. Z. \& Clauset, A. 2014. A unified view of generative models for networks: models, methods, opportunities, and challenges. arXiv preprint arXiv:1411.40\%0.

Janzen, D. H. 1974. Deflowering of central-america. Natural History, 83(4): $48-53$.

Jordano, P. 1987. Patterns of mutualistic interactions in pollination and seed dispersal: connectance, dependence asymmetries, and coevolution. The American Naturalist, 129(5):657-677.

Kahm, M., Hasenbrink, G., Lichtenberg-Frat'e, H., Ludwig, J. \& Kschischo, M. 2010. grofit: Fitting biological growth curves with R. Journal of Statistical Software, 33(7):1-21.

Kaiser-Bunbury, C. N., Muff, S., Memmott, J., Müller, C. B. \& Caflisch, A. 2010. The robustness of pollination networks to the loss of species and interactions: a quantitative approach incorporating pollinator behaviour. Ecology Letters, 13(4):442-452.

Kaiser-Bunbury, C. N., Mougal, J., Whittington, A. E., Valentin, T., Gabriel, R., Olesen, J. M. \& Blüthgen, N. 2017. Ecosystem restoration strengthens pollination network resilience and function. Nature, 542(7640):223-227.

Kalinka, A. T. \& Tomancak, P. 2011. linkcomm: an r package for the generation, visualization, and analysis of link communities in networks of arbitrary size and type. Bioinformatics, 27(14).

Kaneryd, L., Borrvall, C., Berg, S., Curtsdotter, A., Eklöf, A., Hauzy, C., Jonsson, T., Münger, P., Setzer, M., Säterberg, T. et al. 2012. Species-rich ecosystems are vulnerable to cascading extinctions in an increasingly variable world. Ecology and Evolution, 2(4):858-874.

Kauffman, S. A. 1969. Metabolic stability and epigenesis in randomly constructed genetic nets. Journal of Theoretical Biology, 22(3):437-467. 
Kay, K. M. \& Schemske, D. W. 2004. Geographic patterns in plant-pollinator mutualistic networks: comment. Ecology, 85(3):875-878.

Kembel, S., Cowan, P., Helmus, M., Cornwell, W., Morlon, H., Ackerly, D., Blomberg, S. \& Webb, C. 2010. Picante: R tools for integrating phylogenies and ecology. Bioinformatics, 26:1463-1464.

Klein, A., Vaissière, B., Cane, J., Steffan-Dewenter, I., Cunningham, S., Kremen, C. \& Tscharntke, T. 2007. Importance of pollinators in changing landscapes for world crops. Proc. R. Soc. B, 274:303-313.

Koh, L. P., Dunn, R. R., Sodhi, N. S., Colwell, R. K., Proctor, H. C. \& Smith, V. S. 2004a. Species coextinctions and the biodiversity crisis. Science, 305 (5690):1632-1634.

Koh, L. P., Sodhi, N. S. \& Brook, B. W. 2004b. Co-extinctions of tropical butterflies and their hostplants. Biotropica, 36(2):272-274.

Kremen, C. \& M'Gonigle, L. K. 2015. Small-scale restoration in intensive agricultural landscapes supports more specialized and less mobile pollinator species. J. Appl. Ecol., 52:602-610.

Kuznetsova, A., Bruun Brockhoff, P. \& Haubo Bojesen Christensen, R. lmerTest: Tests for random and fixed effects for linear mixed effect models (lmer objects of lme4 package)., 2014. R package version 2.0-11.

Labandeira, C. C., Johnson, K. R. \& Wilf, P. 2002. Impact of the terminal cretaceous event on plant-insect associations. Proceedings of the National Academy of Sciences, 99(4):2061-2066.

Leventhal, G. E., Hill, A. L., Nowak, M. A. \& Bonhoeffer, S. 2015. Evolution and emergence of infectious diseases in theoretical and real-world networks. Nature communications, 6.

Lever, J. J., Nes, E. H., Scheffer, M. \& Bascompte, J. 2014. The sudden collapse of pollinator communities. Ecology Letters, 17(3):350-359.

Lewinsohn, T. M., Inácio Prado, P., Jordano, P., Bascompte, J. \& M Olesen, J. 2006. Structure in plant-animal interaction assemblages. Oikos, 113(1): $174-184$.

Loreau, M., Naeem, S., Inchausti, P., Bengtsson, J., Grime, J., Hector, A., Hooper, D., Huston, M., Raffaelli, D., Schmid, B. et al. 2001. Biodiversity and ecosystem functioning: current knowledge and future challenges. Science, 294(5543):804-808.

MacLeod, M., Genung, M. A., Ascher, J. S. \& Winfree, R. 2016. Measuring 
partner choice in plant-pollinator networks: using null models to separate rewiring and fidelity from chance. Ecology, 97(11):2925-2931.

Manzano, M., Calle, E., Torres-Padrosa, V., Segovia, J. \& Harle, D. 2013. Endurance: A new robustness measure for complex networks under multiple failure scenarios. Computer Networks, 57(17):3641-3653.

Marquitti, F. M. D., Guimarães, P. R., Pires, M. M. \& Bittencourt, L. F. 2014. Modular: software for the autonomous computation of modularity in large network sets. Ecography, 37(3):221-224.

May, R. M. 1971. Stability in multispecies community models. Mathematical Biosciences, 12(1-2):59-79.

May, R. M. 2001. Stability and complexity in model ecosystems, volume 6. Princeton University Press.

McCann, K. 2007. Protecting biostructure. Nature, 446(7131):29-29.

McCann, K. S. 2000. The diversity-stability debate. Nature, 405(6783):228-233.

McCauley, D. J., DeSalles, P. A., Young, H. S., Dunbar, R. B., Dirzo, R., Mills, M. M. \& Micheli, F. 2012. From wing to wing: the persistence of long ecological interaction chains in less-disturbed ecosystems. Scientific Reports, 2:409.

Memmott, J., Waser, N. M. \& Price, M. V. 2004. Tolerance of pollination networks to species extinctions. Proceedings of the Royal Society of London B: Biological Sciences, 271(1557):2605-2611.

Menz, M. H., Phillips, R. D., Winfree, R., Kremen, C., Aizen, M. A., Johnson, S. D. \& Dixon, K. W. 2011. Reconnecting plants and pollinators: challenges in the restoration of pollination mutualisms. Trends in Plant Science, 16(1): $4-12$.

M'Gonigle, L., Ponisio, L., Cutler, K. \& Kremen, C. 2015. Habitat restoration promotes pollinator persistence and colonization in intensively-managed agriculture. Ecol. Appl., 25:1557-1565.

Montoya, D., Rogers, L. \& Memmott, J. 2012. Emerging perspectives in the restoration of biodiversity-based ecosystem services. Trends in Ecology 86 Evolution, 27(12):666-672.

Montoya, J. M., Pimm, S. L. \& Solé, R. V. 2006. Ecological networks and their fragility. Nature, 442(7100):259-264.

Morandin, L. \& Kremen, C. 2013. Hedgerow restoration promotes pollinator populations and exports native bees to adjacent fields. Ecol. Appl., 23:829839. 
Morandin, L., Long, R. \& Kremen, C. 2016. Pest control and pollination costbenefit analysis of hedgerow restoration in a simplified agricultural landscape. Journal of Economic Entomology, pp. tow086.

Naeem, S. \& Li, S. 1997. Biodiversity enhances ecosystem reliability. Nature, 390(6659):507-509.

Newman, M. E. 2002. Spread of epidemic disease on networks. Physical Review E, 66(1):016128.

Newman, M. E. \& Girvan, M. 2004. Finding and evaluating community structure in networks. Physical Review E, 69(2):026113.

Olesen, J. M., Bascompte, J., Dupont, Y. L. \& Jordano, P. 2007. The modularity of pollination networks. Proceedings of the National Academy of Sciences, 104(50):19891-19896.

Olesen, J. M., Bascompte, J., Elberling, H. \& Jordano, P. 2008. Temporal dynamics in a pollination network. Ecology, 89(6):1573-1582.

Ollerton, J. 2006. Plant-pollinator interactions: from specialization to generalization. University of Chicago Press, Chicago. pp. 411-435.

Ollerton, J., Winfree, R. \& Tarrant, S. 2011. How many flowering plants are pollinated by animals? Oikos, 120:321-326.

Paine, R. T. 1966. Food web complexity and species diversity. American Naturalist, pp. 65-75.

Parker, V. T. 1997. The scale of successional models and restoration objectives. Restor. Ecol., 5(4):301-306.

Peel, L. \& Clauset, A. 2014. Detecting change points in the large-scale structure of evolving networks. arXiv preprint arXiv:1403.0989.

Pimm, S. L. 1984. The complexity and stability of ecosystems. Nature, 307 (5949):321-326.

Ponisio, L. C., M'Gonigle, L. K. \& Kremen, C. 2016. On-farm habitat restoration counters biotic homogenization in intensively managed agriculture. Global Change Biol., 22(2):704-715.

Ponisio, L. C., Gaiarsa, M. P. \& Kremen, C. 2017. Opportunistic attachment assembles plant-pollinator networks. Ecology Letters, 48.

Pyke, G. H. 1984. Optimal foraging theory: a critical review. Annu. Rev. Ecol. Evol. Syst., pp. 523-575.

R Core Team. R: A Language and Environment for Statistical Computing. R Foundation for Statistical Computing, Vienna, Austria, 2015. 
R Core Team. R: A Language and Environment for Statistical Computing. R Foundation for Statistical Computing, Vienna, Austria, 2016.

Rezende, E. L., Lavabre, J. E., Guimarães, P. R., Jordano, P. \& Bascompte, J. 2007a. Non-random coextinctions in phylogenetically structured mutualistic networks. Nature, 448(7156):925-928.

Rezende, E. L., Lavabre, J. E., Guimarães, P. R., Jordano, P. \& Bascompte, J. 2007b. Non-random coextinctions in phylogenetically structured mutualistic networks. Nature, 448(7156):925-928.

Rico-Gray, V. \& Oliveira, P. S. 2007. The ecology and evolution of ant-plant interactions. University of Chicago Press.

Rogers, H. S., Buhle, E. R., HilleRisLambers, J., Fricke, E. C., Miller, R. H. \& Tewksbury, J. J. 2017. Effects of an invasive predator cascade to plants via mutualism disruption. Nature Communications, 8:14557.

Rohr, R. P., Saavedra, S. \& Bascompte, J. 2014. On the structural stability of mutualistic systems. Science, 345(6195):1253497.

Saavedra, S., Stouffer, D. B., Uzzi, B. \& Bascompte, J. 2011. Strong contributors to network persistence are the most vulnerable to extinction. Nature, 478(7368):233-235.

Sala, O. E., Chapin, F. S., Armesto, J. J., Berlow, E., Bloomfield, J., Dirzo, R., Huber-Sanwald, E., Huenneke, L. F., Jackson, R. B., Kinzig, A. et al. 2000. Global biodiversity scenarios for the year 2100. Science, 287(5459): 1770-1774.

Santamaría, S., Galeano, J., Pastor, J. M. \& Méndez, M. 2016. Removing interactions, rather than species, casts doubt on the high robustness of pollination networks. Oikos, 125(4):526-534.

Sardiñas, H. S. \& Kremen, C. 2015. Pollination services from field-scale agricultural diversification may be context-dependent. Agriculture, Ecosystems E Environment, 207:17-25.

Sazima, C., Guimarães, P. R., Dos Reis, S. F. \& Sazima, I. 2010. What makes a species central in a cleaning mutualism network? Oikos, 119(8):1319-1325.

Shipley, B. 2004. Cause and correlation in biology: A user's guide to path analysis, structural equations and causal inference. Cambridge University press.

Shirley, M. D. \& Rushton, S. P. 2005. The impacts of network topology on disease spread. Ecological Complexity, 2(3):287-299.

Silva, W., Guimaraes Jr, P., Dos Reis, S., Guimaraes, P. et al. 2007. Investiga- 
ting fragility in plant-frugivore networks: a case study of the atlantic forest in brazil. Seed dispersal: theory and its application in a changing world. Oxfordshire, UK: CAB International, pp. 561-578.

Simon, H. A. 1955. On a class of skew distribution functions. Biometrika, 42: $425-440$.

Sole, R. V. \& Montoya, M. 2001. Complexity and fragility in ecological networks. Proceedings of the Royal Society of London B: Biological Sciences, 268(1480):2039-2045.

Stephens, D. W. \& Krebs, J. R. 1986. Foraging theory. Princeton University Press.

Stouffer, D. B. \& Bascompte, J. 2010. Understanding food-web persistence from local to global scales. Ecology Letters, 13(2):154-161.

Stouffer, D. B. \& Bascompte, J. 2011. Compartmentalization increases foodweb persistence. Proceedings of the National Academy of Sciences, 108(9): 3648-3652.

Suding, K. N. \& Hobbs, R. J. 2009. Threshold models in restoration and conservation: a developing framework. Trends in ecology $\&$ evolution, 24(5): 271-279.

Terborgh, J. \& Estes, J. 2010. Trophic Cascades: predators, prey, and the changing dynamics of nature. Island Press.

Thébault, E. \& Fontaine, C. 2010. Stability of ecological communities and the architecture of mutualistic and trophic networks. Science, 329(5993):853-856.

Thomas, C. D., Cameron, A., Green, R. E., Bakkenes, M., Beaumont, L. J., Collingham, Y. C., Erasmus, B. F., De Siqueira, M. F., Grainger, A., Hannah, L. et al. 2004. Extinction risk from climate change. Nature, 427(6970):145148.

Thompson, J. N. \& Fernandez, C. C. 2006. Temporal dynamics of antagonism and mutualism in a geographically variable plant-insect interaction. Ecology, 87(1):103-112.

Tylianakis, J. M. 2013. The global plight of pollinators. Science, 339(6127): $1532-1533$.

Tylianakis, J. M. \& Morris, R. J. 2017. Ecological networks across environmental gradients. Annu. Rev. Ecol. Evol. Syst., 48.

Tylianakis, J. M., Didham, R. K., Bascompte, J. \& Wardle, D. A. 2008. Global change and species interactions in terrestrial ecosystems. Ecology Letters, 11 (12):1351-1363. 
Tylianakis, J. M., Laliberté, E., Nielsen, A. \& Bascompte, J. 2010. Conservation of species interaction networks. Biological Conservation, 143(10):2270-2279.

Valdovinos, F. S., Brosi, B. J., Briggs, H. M., Moisset de Espanés, P., RamosJiliberto, R. \& Martinez, N. D. 2016. Niche partitioning due to adaptive foraging reverses effects of nestedness and connectance on pollination network stability. Ecology Letters, 19(10):1277-1286.

Valiente-Banuet, A., Aizen, M. A., Alcántara, J. M., Arroyo, J., Cocucci, A., Galetti, M., García, M. B., García, D., Gómez, J. M., Jordano, P. et al. 2015. Beyond species loss: the extinction of ecological interactions in a changing world. Functional Ecology, 29(3):299-307.

Vázquez, D. P. \& Aizen, M. A. 2004. Asymmetric specialization: a pervasive feature of plant-pollinator interactions. Ecology, 85(5):1251-1257.

Vázquez, D. P., Melián, C. J., Williams, N. M., Blüthgen, N., Krasnov, B. R. \& Poulin, R. 2007. Species abundance and asymmetric interaction strength in ecological networks. Oikos, 116(7):1120-1127.

Vázquez, D. P., Blüthgen, N., Cagnolo, L. \& Chacoff, N. P. 2009. Uniting pattern and process in plant-animal mutualistic networks: a review. Ann. of Bot., 103(9):1445-1457.

Vidal, M. M., Hasui, E., Pizo, M. A., Tamashiro, J. Y., Silva, W. R. \& Guimaraes, P. R. 2014. Frugivores at higher risk of extinction are the key elements of a mutualistic network. Ecology, 95(12):3440-3447.

Vieira, M. C. \& Almeida-Neto, M. 2015. A simple stochastic model for complex coextinctions in mutualistic networks: robustness decreases with connectance. Ecology Letters, 18(2):144-152.

Waser, N. M., Chittka, L., Price, M. V., Williams, N. M. \& Ollerton, J. 1996. Generalization in pollination systems, and why it matters. Ecology, 77:1043.

Winfree, R., Williams, N. M., Dushoff, J. \& Kremen, C. 2014. Species abundance, not diet breadth, drives the persistence of the most linked pollinators as plant-pollinator networks disassemble. The American Naturalist, 183(5): 600-611.

Wotton, D. M. \& Kelly, D. 2012. Do larger frugivores move seeds further? body size, seed dispersal distance, and a case study of a large, sedentary pigeon. Journal of Biogeography, 39(11):1973-1983.

Yeakel, J. D., Pires, M. M., Rudolf, L., Dominy, N. J., Koch, P. L., Guimarães Jr., P. R. \& Gross, T. 2014. Collapse of an ecological network in 
ancient egypt. Proceedings of the National Academy of Sciences, 111(40): 14472-14477.

Young, H. S., McCauley, D. J., Galetti, M. \& Dirzo, R. 2016. Patterns, causes, and consequences of anthropocene defaunation. Annu. Rev. Ecol. Evol. Syst., 47:333-358.

Zavaleta, E. S. \& Hulvey, K. B. 2004. Realistic species losses disproportionately reduce grassland resistance to biological invaders. Science, 306(5699):11751177.

Zuur, A. F., Ieno, E. N. \& Elphick, C. S. 2010. A protocol for data exploration to avoid common statistical problems. Methods in Ecology and Evolution, 1 (1):3-14. 
[...] You put the work in, don't worry about the praise, my love; Don't try to change the world, find something that you love and do it every day Do that, for the rest, of your life, And eventually, the world will change.

- Ben Haggerty

You have Brains in your head. You have feet in your shoes. You can steer yourself any direction you choose. 